\title{
Intracranial Germinoma: Atypical Presentation
}

Viviana Calderón-Molina ${ }^{1 *}$, Fernando Alvarado Calderón ${ }^{2}$, Mónica Mohs-Alfaro² and Manuel Hernádez Gaitán ${ }^{3}$

${ }^{1}$ Maximiliano Peralta Jiménez Hospital, Cartago, Costa Rica

${ }^{2}$ Pathology Services, Rafael Ángel Calderón Guardia Hospital, San José, Costa Rica

${ }^{3}$ MR National Center of the Caja Costarricense de Seguro Social, San José, Costa Rica

\begin{abstract}
Fourteen year old female patient referred in June 2013 to the Emergency Department due to seizures of recent onset. The patient had a six-month history of behavioral changes and impaired academic performance; also, days before the seizure episodes she developed vomiting. The physical examination documented bradypsychia and papilledema. The results of hormone testing showed panhypopituitarism, a condition of the hypothalamic-hypophysis axis. An MRI was performed and it showed a heterogeneous tumor that compromises the frontal periventricular white matter extending through the rostrum of the corpus callosum, infiltrates the ependyma, it is heterogeneous with cystic areas; after contrast, it presents heterogeneous enhancement. In univoxel MR spectroscopy, it was observed an increase of choline, lipid and lactate, and a decrease in $\mathrm{N}$-acetyl aspartate. It associates with the existence of edema in the bifrontal white matter, there is mass effect given by the compression of the frontal recesses of the lateral ventricles and effacement of the frontal anterior sulci. There is a second lesion that compromises the tuber cinereum, pituitary stalk and optic chiasm, it enhances after contrast administration and presents cystic areas. For the morphological characteristics and changes in spectroscopy, differential diagnosis as primitive neuroectodermal tumor (PNET) and glioblastoma were planted. The frozen biopsy reports a small celled tumor: PNET vs. Lymphoma. Due to the latter, a complete macroscopic resection is performed via a bifrontal craniotomy with an interhemispheric approach. Diabetes insipidus is developed immediately during the postoperative period. The full section biopsy reported a germinoma. Managed through sequential chemotherapy and radiation therapy.
\end{abstract}

Keywords: Germinoma; Neurosurgery; Neuropathology; Neuroradiology

\section{Introduction}

In germ cell tumors, germinomas account for approximately a $65 \%$, classified as grade III of the WHO [1-3]. Furthermore, they represent a $0.4-3.4 \%$ of brain tumors and an $8-15 \%$ of the pediatric instances. Between $80-90 \%$ of the cases occur in people under 25 years of age, with a peak incidence rate between $10-14$ year olds. They are not commonly diagnosed past the patient's third decade $[3,4]$ and it is most common in male patients. In terms of location, suprasellar is most common in women and in the pineal region for men $[2,3,5,6]$. Most are located towards the midline and more than the $80 \%$ appear in structures above the third ventricle, the pineal gland is being the most common place of origin followed by the suprasellar compartment, for instance, the neurohypophyseal axis, periventricular, intraventricular, lymph nodes, basal ganglia, brain hemispheres, intramedullary, among others. They are most common in the suprasellar and thalamic regions as well as the basal ganglia $[3,5,7]$.

\section{Its etiology is unknown [3].}

Pure germ cell tumors, as the one at hand, when related to the Central Nervous System, are called germinomas, seminomas when they originate in the testicules and dysgerminoma when related to the ovaries. All these share the same histopathological pattern, immunohistochemistry profile and serum markers [1].

\section{Case Report}

Fourteen year old female patient referred in June 2013 to the Emergency Department due to seizures of recent onset. The patient had a six-month history of behavioral changes and impaired academic performance; also, days before the seizure episodes she developed vomiting. The patient denies suffering from any chronic pathology. The physical examination documented bradypsychia and papilledema. The results of hormone testing showed panhypopituitarism, a condition of the hypothalamic-hypophysis axis. An MRI was performed and it showed a heterogeneous tumor that compromises the frontal periventricular white matter extending through the rostrum of the corpus callosum, infiltrates the ependyma, it is heterogeneous with cystic areas; after contrast, it presents heterogeneous enhancement. In univoxel MR spectroscopy, it was observed an increase of choline, lipid and lactate, and a decrease in $\mathrm{N}$-acetyl aspartate. It associates with the existence of edema in the bifrontal white matter, there is mass effect given by the compression of the frontal recesses of the lateral ventricles and effacement of the frontal anterior sulci. There is a second lesion that compromises the tuber cinereum, pituitary stalk and optic chiasm, it enhances after contrast administration and presents cystic areas. For the morphological characteristics and changes in spectroscopy, differential diagnosis as primitive neuroectodermal tumor and glioblastoma were planted. Anticonvulsant and steroid therapy was initiated. The results of hormone testing show a decrease in T4, T3, TSH, LH, FSH, GRH and cortisol, panhypopituitarism, the hypothalamic-hypophysis axis is compromised. During surgery, a frozen biopsy is performed, which indicates a small-cell tumor: PNET vs. lymphoma. Due to the latter, a complete macroscopic resection is performed via a bifrontal craniotomy with an interhemispheric approach. Diabetes insipidus is developed immediately during the postoperative period and treated with DDAVP. An iliac crest biopsy is performed as well as a bone marrow aspiration,

*Corresponding author: Viviana Calderón-Molina, General Medicine Practitioner Maximiliano Peralta Jiménez Hospital, Cartago, Costa Rica, Tel: 506-83324479; E-mail: vivicalmo@gmail.com

Received January 09, 2015; Accepted March 09, 2015; Published March 11 2015

Citation: Calderón-Molina V, Calderón FA, Mohs-Alfaro M, Gaitán MH (2015) Intracranial Germinoma: Atypical Presentation. J Neurol Disord 3: 215. doi:10.4172/2329-6895.1000215

Copyright: (c) 2015 Calderón-Molina V. This is an open-access article distributed under the terms of the Creative Commons Attribution License, which permits unrestricted use, distribution, and reproduction in any medium, provided the original author and source are credited. 
Citation: Calderón-Molina V, Calderón FA, Mohs-Alfaro M, Gaitán MH (2015) Intracranial Germinoma: Atypical Presentation. J Neurol Disord 3: 215. doi:10.4172/2329-6895.1000215

both reporting no neoplastic infiltration. Studies of the Cerebrospinal fluid (CSF) result negative for tumor markers, but positive for the presence of neoplastic cells (Figures 1-6).

A Suprasellar Germinoma is therefore diagnosed.

A postoperative CT scan showed no residual lesion, hence metastatic disease was ruled out in thorax and abdomen.

Based on these findings, the decision is to proceed with sequential chemotherapy in a four-cycle schedule of BEP (Bleomycin, Etoposide and Cisplatin) and radiation of the neural axis in a dose of $45 \mathrm{~Gy}$ in cranial and $30.6 \mathrm{~Gy}$ in the spinal fields.

As of January 2015, the patient is in excellent clinical condition with pharmacological treatment with Levothyroxine, DDAVP and hydrocortisone. The LCR cytology continues to result negative for malignancy and with no evidence or relapse upon imaging.

\section{Discussion}

\section{Clinical discussion}

By affecting the pineal region, a compression and obstruction of the cerebral aqueduct is produced, causing hydrocephalus as well

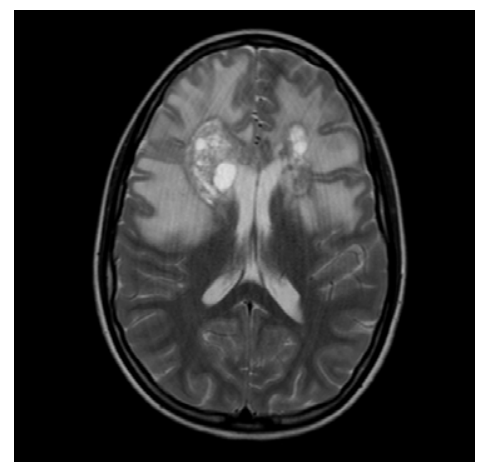

Figure 1: A bilateral frontal periventricular tumor with extension through the rostrum of the corpus callosum is observed, presents microcysts and behaves hypointense in T2 weighted imaging, produces mass effect compressing the frontal recesses of the lateral ventricles and associates the existence of bilateral frontal perilesional edema.

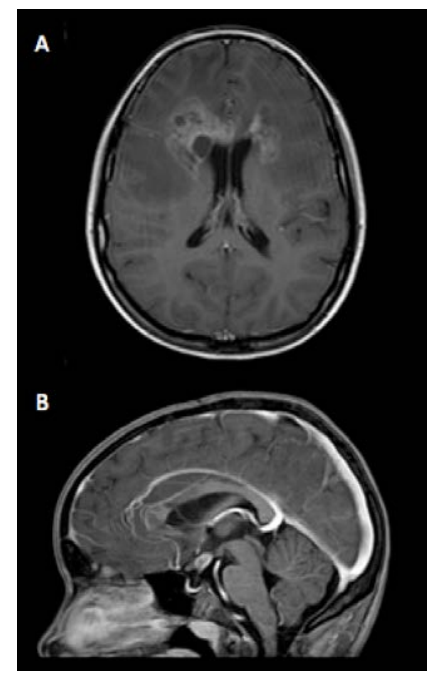

Figure 2: A) heterogeneous enhancement of the bilateral frontal tumor, B) the hypothalamic tumor that extends to the pituitary stalk.

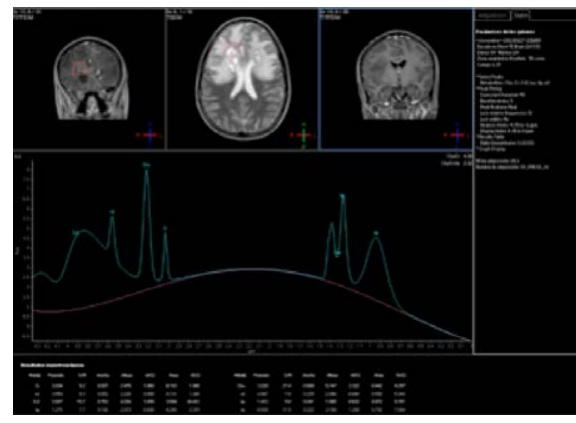

Figure 3: Right frontal periventricular univoxel MR spectroscopy where it is observed increased choline and lactate and decreased $\mathrm{N}$ - acetyl aspartate.

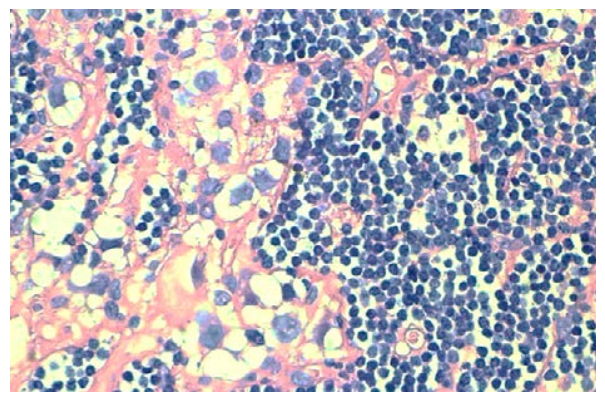

Figure 4: Histological features of germinoma. Tumor cells surrounded by fibro vascular septae and lymphocytic T cells.

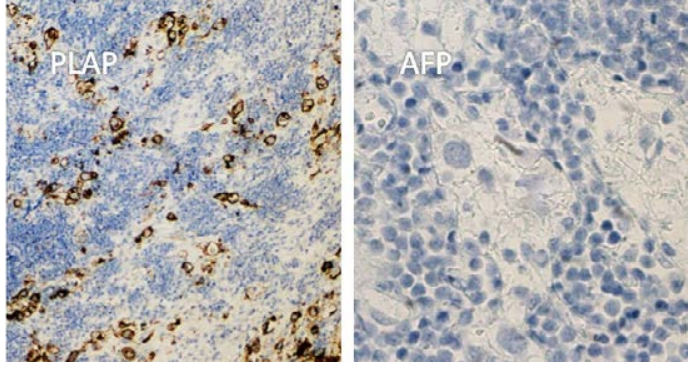

Figure 5: Immunohistochemistry expression. Tumor cells with membranous and cytoplasmatic for PLAP. Staining reaction is absent to AFP.

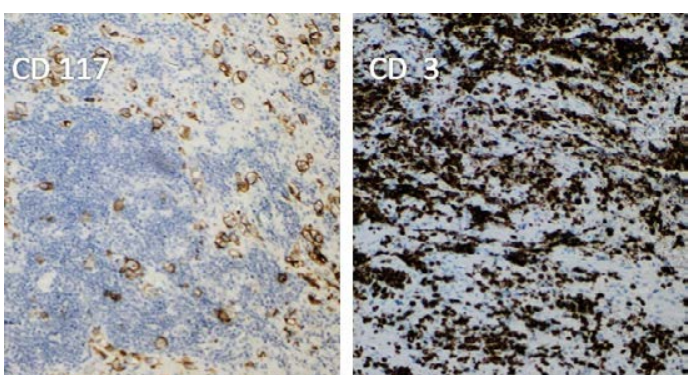

Figure 6: Immunohistochemistry expression. Tumor cells with expression of CD 117 (c-kit) and expression of CD 3 lymphocytes.

as intracranial hypertension, Parynaud syndrome when the rostral pretectal area and the superior colliculus are compressed; also, deafness when compressing the inferior colliculus, delayed growth, sexual maturation, early puberty, behavioral changes, and impaired academic performance. 
A classic triad is determined when the suprasellar region is affected, when there are visual field defects, diabetes insipidus and panhypopituitarism. Further metastases seedings through the CSF can result in radiculopathy and myelopathy $[2,3,5-8]$. Reports show that many patients state to suffer from memory loss; a retrospective study shows that a $42 \%$ incidence rate of significant amnesia was observed in older patients within the typical age group. No relation was found between hydrocephalus at the time of diagnosis, radiation exposure or involvement of the classic memory structures; which suggests that there is a significant risk of amnesia between these patients [9-11].

\section{Diagnosis}

Neuroimaging: Neuroimaging is nonspecific and usually appearing as iso and hyperdense solid masses relative to gray matter and enhanced by contrast. From isointense to low intensity on T1 and hyperintense on T2; after treatment, the tumor can become hypodense and brighter on $\mathrm{T} 2$ and FLAIR. Intratumoral hemorrhage can be found in germinomas with elevated B- human chorionic gonadotropin (B-hCG) $[3,5,6,9]$.

Tumor markers: Findings of elevated AFP on yolk sac-derived tumors in moderate amount may indicate the expression of enteric components of a teratoma [3]. High elevations of B-hCG suggest choriocarcinoma components; more modest elevations may be associated with germinomas that may contain syncytiotrophoblastic elements $[2,3,5,9]$. PLAP elevations correlate to a pure germinomatous tumor [3].

It is also of great use when evaluating the patient's response to treatment and early detection of relapse $[8,9]$.

The absence of germ cell markers should be interpreted with caution, as it does not rule out the presence of germinoma or embryonal cell carcinoma [9].

Macroscopically, it is usually solid, but may still contain small cystic lesions, and is composed of soft and friable white-dark tissue $[1,3]$. There is usually no hemorrhage or necrosis, however, if present, it suggests an increase of malignant components [3].

The histopathology is critical to the diagnosis, and thus, to consider the treatment and prognosis $[3,5,9]$.

Intracranial germ cell tumors are usually of mixed composition, except germinoma and teratoma [3].

A germinoma is composed of large cells that appear undifferentiated, and resemble primordial germ elements. They are arranged in monomorphic sheets, lobes, characterized by a stromal response, organized in cords or trabeculae. It is identified by a round vesicular central nucleus, prominent nucleoli, discrete cell membranes and clear abundant cytoplasm due to the accumulation of glycogen. It is common to identify mitotic figures, but rarely necrosis. With some frequency, delicate fibro vascular septa, mainly infiltrated by $\mathrm{T}$ lymphocytes, are also found. Some germinomas show a granulomatous reaction, which can be confused with sarcoidosis or tuberculosis. Others have extensive fibrous tissue. As for immunohistochemistry, a strong labeling of membrane by CD117 (c-kit), Sal-like 4 (SALL4) and Octamen binding transcription-3/4 (OCT3/4) nuclear reactivity are more constant (the last two are not available in our country). The cytoplasmic and membrane staining for PLAP is less common, as well as difficult to demonstrate in inflammatory and previously frozen tissue. A minority shows patchy foci labeling for cytokeratins in cytoplasm. Typical germinomas may contain giant syncytiotrophoblastic cells that manifest in their cytoplasm B-hCG, human placental lactogen (HLP) and cytokeratins $[1,3,5,6,9,12,13]$.

\section{Histogenesis}

They are accepted to represent the progeny of primordial germ cells that migrated aberrantly or with purpose to the embryonic CNS instead of developing in the genital ridges. They have been specifically defined as an enigmatic population of skeletal muscle cells native to the development of the pineal gland, proposed as possible descendants of primitive germ elements attracted to this organ during neuroembriogenesis [3].

Alternative to this hypothesis is the proposition of its origin in a variety of displaced embryonic tissue and incorporated into the developing neural tube, in which case, only the germinoma would derive from the lost primordial germ cells, hence qualifying as a true germ cell neoplasm [3].

Another theory involves toti- or pluri-potent stem cells [3].

The differences between these types of tumors may reflect different creation mechanisms instead of divergent cellular origins [3].

Investigations with regards to the molecular biology involved in the pathogenesis have been initiated. Mutations in KIT, KRAS/ NRAS as well as caspase B-lineage lymphoma (CBL) have been described [8].

\section{Prognosis}

The predictive factor of most value is the histological subtype. Pure germinomas are extremely radiosensitive $[1,3,6,9,11,14]$. The five-year survival rate is estimated to over $75 \%$ and the ten-year rate to $69 \%$ with radiation alone, adding chemotherapy can improve disease control by reducing the radiation dose. Germinomas with syncytiotrophoblast cells or that are associated with elevated B-hCG levels have reported a higher risk of local failure, and a modest decrease in survival $[3,9,15]$.

The typical patterns of progression for this disease are local recurrence and dissemination through the CSF, abdominal contamination via ventriculoperitoneal shunts and hematogenous spread, mainly to lungs and bones [1-3].

\section{Management}

Performance of serum and CSF (B-hCG, AFP, PLAP) $[2,8,16]$. Tumor markers as well as CSF cytology $[5,15]$ is recommended.

MRI of the neuraxis (cervical, thoracic, lumbar) for metastatic seeding $[2,5,8,16]$.

\section{Lesion biopsy $[2,8,16]$}

Chemotherapy and radiation therapy $[2,8]$. In the final report of the SIOP CNS GCT 96 study, multinational, prospective, nonrandomized, in which single craniospinal irradiation was compared with chemotherapy followed by focal irradiation to the primary site, it was concluded that localized germinomas can be treated with reduced craniospinal radiation alone or with chemotherapy and radiation in a reduced field. There was no difference in the overall five-year survival rate, but a difference was noted in terms of progression-free survival; also, the relapse pattern suggests that the ventricles must be included in the radiation field, and that a low dose craniospinal irradiation alone is effective in metastatic disease $[5,17,18]$. Multinational experimental studies are currently being conducted to perform bone marrow or peripheral stem cell transplants in order to use higher doses of chemotherapy and/or radiation [19]. 
Total resection of the lesion is usually not recommended because of the risk of surgical complications and the fact that germinomas are affectedly sensitive to radiation; the latter unless the patient's clinical stability is compromised [5].

If there is a case of hydrocephalus, external ventricular drainage is recommended in order to prevent peritoneal seeding, this, despite the uncommonness of its presentation, and that after the treatment of their tumor, the patient will probably not need a permanent device $[2,5,8]$.

Clinical monitoring is recommended for 10 years and imaging for at least 5 years. If there were positive tumor markers, the recommendation is to follow up with markers as well [8].

\section{Conclusion}

As with all patients, it is important to make use of all the resources available in order to make an accurate diagnosis considering that the prognosis and quality of life of the patients depend on it. This case was challenging in terms of diagnosis since it was atypical both clinically and radiologically.

\section{References}

1. Ellison D, Love S, Chimelli L (2013) Neuropathology: A Reference text of CNS pathology. (3rdedn), Elsevier.

2. Greenberg Mark S (2010) Handbook of Neurosurgery. (7thedn) Thieme Medical Publishers. Chapter 21. Tumor 691-695.

3. Louis David N, Ohgaki H, Wrestler OD, Cave Nee WK (2007) WHO classification of tumors of the central nervous system. (4thedn), International Agency for Research on Cancer (IARC). Lyon.

4. Helmut B, Niklaus K, Wess (2011) Ventricular Tumors. In: Youmans Neurological Surgery (6thedn), Elsevier Saunders. Philadelphia, United States.

5. Jack S (2014) Intracraneal germ cell tumors. Wolters Kluwer editorial. Acceso a través de SIBDI.

6. Anthony Y, Marie RZ (2014) Germ cell tumors of the Central Nervous System. Neuropathology, Saunders, Elsevier.
7. Henry B, Raymond S, Chiocca E Antonio (2011) Brain Tumors: An Overview of Current Histopathologic Classifications. In: Youmans Neurological Surgery (6thedn), Elsevier Saunders. Philadelphia, United States.

8. Anthony P, Meriel J (2014) Germ cell tumours in children and adolescents What's new? Practice points. Paediatrics and Child Health. Elsevier. 24: 148154

9. Bruce Jeffrey N (2011) Pineal Tumors. In: Youmans Neurological Surgery (6thedn), Elsevier Saunders. Philadelphia, United States.

10. Hill P, Lepre F (1994) CNS germinomas: curable tumours in two adolescents. Journal Paediatrics and Child Health. 30: 68-71.

11. Wilkening GN, Madden JR (2011) Memory déficits in patients with pediatric CNS germ cell tumors. Pediatrics Blood Cancer 57: 486-491.

12. Michael K, Nafi A, David Y (2011) Radiologic Features of Central Nervous System Tumors. In: Youmans Neurological Surgery. (6thedn), Elsevier Saunders. Philadelphia, United States.

13. Francoise G, Cahrles D, De Girolami Umberto (2014), Escourolle and Poirier's. Manual of Basic Neuropathology.(5thedn), Oxford university press.

14. Hadziahmetovic M, Clarke JW (2008) CNS germinomas: what is the best treatment strategy? Expert Rev Neurother 8: 1527-1536.

15. McCarthy BJ, Shibui S (2012) Primary CNS germn cell tumors in Japan and the United States: an analysis of 4 tumor registries. Neuro-oncology 14: 11941200 .

16. Bruce Jeffrey N (2011) Pineal Tumors. In: Youmans Neurological Surgery (6thedn), Elsevier Saunders. Philadelphia, United States.

17. Calaminus G, Kortmann R (2013) SIOP CNS GCT 96: final report of outcome of a prospective, multinational nonrandomized trial for children and adults with intracranial germinoma, comparing craniospinal irradiation alone with chemotherapy followed by focal primary site irradiation for patients with localized disease. Neuro-oncology15: 788-796.

18. Massao M, Yukitaka U (1998) Germ cell tumors of the central nervous system Combined chemotherapy and radiation therapy for central nervous system germ cell tumors: preliminary results of a Phase II study of the Japanese Pediatric Brain Tumor Study Group. Neurosurgical Focus. The JNS Org 5: 18.

19. http://clinicaltrials.gov/show/NCT00025324 